\title{
Single-level awake transforaminal lumbar interbody fusion: a Mayo Clinic institutional experience and national analysis
}

\author{
Kingsley Abode-lyamah, MD, ${ }^{1}$ Abdul Karim Ghaith, MD, ${ }^{2,3}$ Archis R. Bhandarkar, BS, ${ }^{2,3}$ \\ Gaetano De Biase, MD, ${ }^{1}$ Rami Rajjoub, ${ }^{2,3}$ Selby G. Chen, MD, ${ }^{1}$ Alfredo Quiñones-Hinojosa, MD, ${ }^{1}$ \\ and Mohamad Bydon, MD2,3 \\ 1Department of Neurosurgery, Mayo Clinic, Jacksonville, Florida; ${ }^{2}$ Department of Neurosurgery, Mayo Clinic, Rochester; and
${ }^{3}$ Neuro-Informatics Laboratory, Mayo Clinic, Rochester, Minnesota
}

\begin{abstract}
OBJECTIVE Awake transforaminal lumbar interbody fusion (TLIF) is a novel technique for performing spinal fusions in patients under conscious sedation. Whether awake TLIF can reduce operative times and decrease the hospital length of stay (LOS) remains to be shown. In this study, the authors sought to assess the differences in clinical outcomes between patients who underwent awake TLIF and those who underwent TLIF under general anesthesia by using institutional experience at the Mayo Clinic and the National Surgical Quality Improvement Program (NSQIP) database.
\end{abstract}

METHODS Chart review was performed for a consecutive series of patients who underwent single-level minimally invasive surgery (MIS)-TLIF performed by a single surgeon (K.A.I.) at a single institution. Additionally, the NSQIP database was queried from 2016 to 2019 for patients who underwent awake TLIF as well as propensity score-matched patients who underwent TLIF under general anesthesia.

RESULTS A total of 20 patients at Mayo Clinic underwent awake single-level MIS-TLIF. The mean operative time was $122 \pm 16.68$ minutes, and the mean estimated blood loss was $39 \pm 30.24 \mathrm{ml}$. No intraoperative complications were reported. A total of 96 patients who underwent TLIF (24 awake and 72 under general anesthesia) were analyzed from the NSQIP database. The mean LOS was less in the awake cohort (1.4 \pm 1.381 days) than the general anesthesia cohort ( 3 \pm 2.274 days) $(p=0.002)$.

CONCLUSIONS Evidence from the authors' institutional experience and national analysis has demonstrated that awake MIS-TLIF is efficient and can reduce hospital LOS.

https://thejns.org/doi/abs/10.3171/2021.9.FOCUS21457

KEYWORDS awake; anesthesia; minimally invasive; transforaminal lumbar interbody fusion; TLIF; spine

$\mathrm{T}$ RANSFORAMINAL lumbar interbody fusion (TLIF) is a surgical procedure that aims to correct spine alignment and alleviate pain symptoms from degenerative processes that affect the spine. ${ }^{1}$ The TLIF approach involves accessing the intervertebral space through a unilateral route, usually followed by bone grafting and cage insertion. ${ }^{2}$ Although it has been proven to be a safe and effective technique for lumbar fusion, TLIF has been associated with substantial morbidity due to the extensive muscle dissection and retraction required in the surgical procedure. ${ }^{3}$ For instance, prolonged pressure exerted from the retractor blades onto the paraspinal muscles have been reported to diminish trunk muscle strength and induce postoperative skeletomuscular injuries. ${ }^{4}$ To counteract these disadvantages, Foley et al. pioneered the minimally invasive surgery (MIS)-TLIF technique. ${ }^{5}$ Recent literature has demonstrated positive outcomes for patients undergoing MIS-TLIF, including minimized operative time, reduced postoperative analgesic use, decreased hospital length of stay (LOS), and less overall soft-tissue damage. ${ }^{6}$

Patients undergoing MIS-TLIF are typically intubated and administered general anesthesia; however, the perioperative adverse effects of general anesthesia such as memory loss, nausea, and transient impairment of sensory and motor functions have caused concerns for patients and physicians.? To address these limitations, several sur-

ABBREVIATIONS ASA = American Society of Anesthesiologists; $\mathrm{COPD}=$ chronic obstructive pulmonary disease; $\mathrm{EBL}=$ estimated blood loss; $\mathrm{LOS}=$ length of stay; $\mathrm{MIS}=$ minimally invasive surgery; NSQIP = National Surgical Quality Improvement Program; PRO = patient-reported outcome; TLIF = transforaminal lumbar interbody fusion. SUBMITTED July 30, 2021. ACCEPTED September 14, 2021.

INCLUDE WHEN CITING DOI: 10.3171/2021.9.FOCUS21457. 
geons have chosen to perform awake TLIF spinal fusions in select patients. Awake MIS-TLIF protocols combine the benefits of both MIS and awake procedures including minimal tissue damage and reduced exposure to anesthetic agents. ${ }^{8}$ However, it remains to be shown whether awake MIS-TLIF can reduce operative times and decrease LOS.

The purpose of this study was to assess the differences in clinical outcomes between patients undergoing awake TLIF and those undergoing TLIF with general anesthesia by assessing our institutional experience at Mayo Clinic, as well as national data from the American College of Surgeons National Surgical Quality Improvement Program (NSQIP) registry.

\section{Methods \\ Institutional Cohort}

Chart review was conducted for a consecutive series of patients who underwent single-level MIS-TLIF performed by a single surgeon (K.A.I.) from February 2020 to October 2020. Age, sex, race, BMI, American Society of Anesthesiologists (ASA) class, and preoperative comorbidities were collected for each patient. Additionally, we report outcomes, including procedure time, LOS, estimated blood loss (EBL), and discharge disposition.

Despite its prospective advantages, awake MIS-TLIF is not appropriate for all surgical candidates. Some patient selection criteria should be taken into consideration when performing this operation. We recommend choosing patients without significant obesity or obstructive sleep apnea, as the need for an urgent intubation might be challenging if excessive $\mathrm{CO}_{2}$ accumulates during the surgery. In addition, patients with claustrophobia or comorbid anxiety may not tolerate the conscious sedation required for the procedure. Those patients may feel uncomfortable during the surgery because of the long periods of prone positioning and the sounds of surgical instruments, which may elevate the heart rate and/or blood pressure.

At our institution, we used $12.5 \mathrm{mg}$ to $15 \mathrm{mg}$ of isobaric intrathecal bupivacaine with or without the addition of intrathecal narcotic (fentanyl or hydromorphone). The drug was administered one level above the level of planned surgical intervention. Prior to administration of the spinal anesthesia, fentanyl and/or $2 \mathrm{mg}$ of midazolam (100 $\mu \mathrm{g})$ were administered. Intraoperative medication such as dexmedetomidine $(0.1-0.2 \mu \mathrm{g} / \mathrm{kg}$ per hour intravenously, as needed), propofol (10-25 $\mu \mathrm{g} / \mathrm{kg}$ per minute intravenously, as needed), midazolam, or fentanyl was administered to provide patient comfort during the operation.

\section{National Analysis}

The NSQIP database was queried from 2016 to 2019 for all elective procedures with a principal Current Procedural Terminology (CPT) code of 22612 ("arthrodesis, posterior or posterolateral technique, single level; lumbar with lateral transverse technique, when performed"). Similar to the study by Garcia et al., ${ }^{9}$ who used the NSQIP database to study readmissions after TLIF, we excluded procedures that did not use a transverse lateral approach or involved more than one level by excluding admissions that contained additional CPT codes, such as 22614, 22630,
22632, 22633, and 22634. The "ANESTHES" variable in the NSQIP database was used to determine the anesthesia type. We considered procedures with an anesthesia category of epidural, local, monitored anesthesia care/intravenous sedation, regional, or spinal anesthesia as awake procedures. Awake patients were matched against patients who had surgery under general anesthesia using 1:3 propensity score matching based on age, sex, race, ASA class, functional status, and comorbidities (diabetes, chronic obstructive pulmonary disease [COPD], heart failure, dyspnea, and hypertension requiring medication).

All statistical analyses were performed using $\mathrm{R}$ version 4.1.0 (The R Project). Propensity score matching was performed using the matchit() function from the "MatchIt" package. Univariate analysis comparing awake cases versus matched general anesthesia cases was performed using the tableby() function from the "arsenal" package. All continuous outcomes were compared using ANOVA tests, and categorical outcomes were compared using the chi-square test.

\section{Results}

\section{Institutional Cohort}

A total of 20 patients ( 8 men and 12 women) underwent single-level MIS-TLIF awake spinal fusion at Mayo Clinic. The mean age \pm SD of the included patients was 63.6 \pm 8.25 years. The mean BMI was $30.76 \pm 4.83 \mathrm{~kg} / \mathrm{m}^{2}$. The level of fusion was L4-5 in 11 patients (55\%), L5-S1 in 5 patients (25\%), L4-S1 in 2 patients (10\%), L3-5 in 1 patient $(5 \%)$, and L3-4 in 1 patient (5\%). Most patients who underwent awake MIS-TLIF were female $(\mathrm{n}=12,60 \%)$, and the majority were White $(\mathrm{n}=19,95 \%)$, with 1 African American patient (5\%) included. The patient demographic characteristics are presented in Table 1.

Of all 20 patients, 12 presented with preoperative comorbidities, with 9 patients presenting with $\geq 2$ simultaneous comorbidities. Among the comorbidities, hypertension $(\mathrm{n}=9,45 \%)$ and obesity $(\mathrm{n}=9,45 \%)$ were the most prevalent, followed by diabetes mellitus ( $n=4,20 \%)$, sleep apnea $(\mathrm{n}=4,20 \%)$, and coronary artery disease $(\mathrm{n}=2$, $10 \%$ ) (Table 2).

In terms of surgical characteristics, 11 patients (55\%) presented with a preoperative ASA class of II, while the remaining 9 patients (45\%) presented with an ASA class of III. The mean operative time was 122 minutes, ranging from 98 to 162 minutes. The mean EBL was $39 \mathrm{ml}$, ranging from 10 to $150 \mathrm{ml}$. The mean LOS was $1.15 \pm$ 1.25 days, with 7 patients discharged on the same day. All patients were successfully discharged home. The surgical characteristics of all patients are listed in Table 3.

\section{National Analysis}

A total of 96 patients were included from the NSQIP database. The mean age of patients was $57.9 \pm 14.1$ years. The majority of patients were male $(\mathrm{n}=65,67.7 \%)$ and predominately White $(\mathrm{n}=93,96.9 \%)$. There was no statistical significance between the awake and general anesthesia groups in terms of age, sex, race, and comorbidities (including diabetes, COPD, and hypertension) after matching (Table 4).

The general anesthesia group included 72 patients with 
TABLE 1. Demographic characteristics of patients who underwent awake spinal fusion

\begin{tabular}{|c|c|c|c|c|c|}
\hline Pt No. & Age at Surgery (yrs) & Procedure & BMI & Sex & Race \\
\hline 1 & 66 & MIS L4-5 TLIF & 19.2 & $\mathrm{~F}$ & White \\
\hline 2 & 53 & MIS L5-S1 TLIF & 31.5 & M & White \\
\hline 3 & 55 & MIS L4-5 TLIF & 34.6 & $\mathrm{~F}$ & African American \\
\hline 4 & 51 & MIS L5-S1 TLIF & 29.3 & $\mathrm{~F}$ & White \\
\hline 5 & 77 & MIS L4-5 TLIF & 32.8 & $\mathrm{~F}$ & White \\
\hline 6 & 65 & MIS L4-5 TLIF & 29.5 & $\mathrm{~F}$ & White \\
\hline 7 & 78 & MIS L3-5 TLIF & 24.7 & M & White \\
\hline 8 & 84 & MIS L5-S1 TLIF & 34 & M & White \\
\hline 9 & 57 & MIS L4-5 TLIF & 23.9 & $\mathrm{~F}$ & White \\
\hline 10 & 84 & MIS L4-S1 TLIF & 30.2 & M & White \\
\hline 11 & 64 & MIS L5-S1 TLIF & 33.4 & M & White \\
\hline 12 & 57 & MIS L4-5 TLIF & 23.9 & $\mathrm{~F}$ & White \\
\hline 13 & 62 & MIS L4-5 TLIF & 32.8 & M & White \\
\hline 14 & 60 & MIS L4-5 TLIF & 37.7 & $M$ & White \\
\hline 15 & 61 & MIS L4-S1 TLIF & 38.5 & $\mathrm{~F}$ & White \\
\hline 16 & 65 & MIS L4-5 TLIF & 31.9 & $\mathrm{~F}$ & White \\
\hline 17 & 50 & MIS L5-S1 TLIF & 34.9 & $\mathrm{~F}$ & White \\
\hline 18 & 70 & MIS L4-5 TLIF & 27.9 & M & White \\
\hline 19 & 56 & MIS L3-4 TLIF & 26.5 & $\mathrm{~F}$ & White \\
\hline 20 & 56 & MIS L4-5 TLIF & 37.9 & $\mathrm{~F}$ & White \\
\hline
\end{tabular}

$\mathrm{Pt}=$ patient.

TABLE 2. Comorbidities of patients who underwent awake spinal fusion

\begin{tabular}{rcccccc}
\hline Pt & Age at & \multicolumn{5}{c}{ Preop Comorbidities } \\
\cline { 3 - 6 } No. & Surgery (yrs) & CAD & DM & HTN & Obesity & Sleep Apnea \\
\hline 1 & 66 & No & No & No & No & No \\
\hline 2 & 53 & Yes & Yes & Yes & Yes & No \\
\hline 3 & 55 & No & No & No & Yes & No \\
\hline 4 & 51 & No & Yes & Yes & No & No \\
\hline 5 & 77 & No & No & No & No & No \\
\hline 6 & 65 & No & No & No & No & No \\
\hline 7 & 78 & No & No & Yes & No & No \\
\hline 8 & 84 & No & No & Yes & Yes & No \\
\hline 9 & 57 & No & No & No & No & No \\
\hline 10 & 84 & No & No & Yes & No & No \\
\hline 11 & 64 & Yes & Yes & Yes & Yes & Yes \\
\hline 12 & 57 & No & No & No & No & No \\
\hline 13 & 62 & No & No & No & Yes & No \\
\hline 14 & 60 & No & No & Yes & Yes & No \\
\hline 15 & 61 & No & No & No & Yes & Yes \\
\hline 16 & 65 & No & Yes & No & Yes & No \\
\hline 17 & 50 & No & No & No & No & No \\
\hline 18 & 70 & No & No & Yes & No & Yes \\
\hline 19 & 56 & No & No & No & No & Yes \\
\hline 20 & 56 & No & No & Yes & Yes & No \\
\hline
\end{tabular}

$\mathrm{CAD}=$ coronary artery disease DM = diabetes mellitus; HTN = hypertension. 
TABLE 3. Surgical characteristics of patients who underwent awake spinal fusion

\begin{tabular}{|c|c|c|c|c|c|c|}
\hline Pt No. & Age at Surgery (yrs) & ASA Class & Procedure Time (mins) & $\mathrm{EBL}(\mathrm{ml})$ & LOS (days) & Discharge to Home \\
\hline 1 & 66 & II & 121 & 40 & 2 & Yes \\
\hline 2 & 53 & III & 109 & 15 & 1 & Yes \\
\hline 3 & 55 & III & 148 & 75 & 1 & Yes \\
\hline 4 & 51 & III & 125 & 10 & 2 & Yes \\
\hline 5 & 77 & III & 98 & 25 & 1 & Yes \\
\hline 6 & 65 & II & 114 & 25 & 3 & Yes \\
\hline 7 & 78 & II & 146 & 30 & 2 & Yes \\
\hline 8 & 84 & III & 112 & 30 & 1 & Yes \\
\hline 9 & 57 & II & 115 & 30 & 1 & Yes \\
\hline 10 & 84 & II & 135 & 25 & 1 & Yes \\
\hline 11 & 64 & III & 125 & 50 & 2 & Yes \\
\hline 12 & 57 & II & 115 & 30 & 1 & Yes \\
\hline 13 & 62 & II & 109 & 50 & 0 & Yes \\
\hline 14 & 60 & II & 130 & 50 & 5 & Yes \\
\hline 15 & 61 & III & 162 & 150 & 0 & Yes \\
\hline 16 & 65 & II & 127 & 50 & 0 & Yes \\
\hline 17 & 50 & II & 113 & 25 & 0 & Yes \\
\hline 18 & 70 & III & 132 & 25 & 0 & Yes \\
\hline 19 & 56 & II & 101 & 20 & 0 & Yes \\
\hline 20 & 56 & III & 103 & 25 & 0 & Yes \\
\hline
\end{tabular}

a mean age of $57.8 \pm 13.9$ years. Most of these patients were male $(n=49,68.1 \%)$ and White $(n=70,97.2 \%)$. Of those, 6 patients $(8.3 \%)$ presented with insulin-dependent diabetes, 4 patients $(5.6 \%)$ had a history of COPD, and 53 patients $(73.6 \%)$ had a history of hypertension. The awake group included 24 patients with a mean age of $58.3 \pm 14.8$ years. Most of these patients were male $(\mathrm{n}=16,66.7 \%)$ and White $(\mathrm{n}=23,95.8 \%)$. Of those, 2 patients $(8.3 \%)$ presented with insulin-dependent diabetes, 2 patients (8.3\%) had a history of COPD, and 16 patients (66.7\%) had a history of hypertension.

The mean LOS for the awake group was 1.4 days compared with 2.986 days for the general anesthesia group, and the mean operative time was 143.1 hours for the awake group compared with 175.47 hours for the general anesthesia group. Most of the patients who underwent an awake spinal fusion were discharged home (87.5\%) with the remaining patients discharged to a rehabilitation center and skilled care facility ( $4.2 \%$ and $8.3 \%$, respectively). No patients from the awake cohort were readmitted within 30 days following the surgery compared with 3 patients $(4.2 \%)$ who underwent general anesthesia. The mean overall LOS was 2.6 days, with a statistically significant difference between the awake and general anesthesia groups (3.0 vs 1.4 days). There was no statistical significance between the 2 groups in terms of operative time, discharge, and 30-day readmission (Table 4).

\section{Discussion}

Awake MIS spinal fusion protocols are an emerging alternative to traditional spinal surgeries performed un- der general anesthesia or with open techniques. ${ }^{6}$ While many have hypothesized that awake MIS techniques may offer benefits to patients such as decreased postanesthesia recovery periods and reduced LOS, this has not yet been thoroughly evaluated in the literature..$^{10}$ In the current study, we have reported our institutional experience with performing awake MIS-TLIF and also presented a national analysis of recent TLIF cases from the NSQIP registry comparing those performed awake versus those performed under general anesthesia. Our study provides evidence that awake MIS-TLIF can be performed safely with minimal postprocedural complications and reasonable operative times, averaging 122 minutes. Additionally, our national data provide evidence that awake TLIF is associated with decreased hospital LOS compared with TLIF performed under general anesthesia.

Of note, based on our national data on awake TLIF, we did not find that awake TLIF was associated with decreased operative times or reduced readmission rates. This finding could potentially be explained by increased variability in national data from the NSQIP registry compared with our own institutional experience. In our cohort, the range in operative time for awake cases was 96 to 162 minutes. In contrast, the awake TLIF group from the NSQIP database had a range in operative time of 45 to 410 minutes versus a range of 25 to 864 minutes in the matched general anesthesia control group. The significant variability in the NSQIP data may be explained by differing surgeon expertise between centers or differences in reporting quality. This limited our ability to resolve differences in operative times and readmission rates between awake versus general anesthesia cases in our national co- 
TABLE 4. Univariate analysis comparing patients who received awake spine surgery with propensity score-matched control patients who received general anesthesia from the NSQIP database

\begin{tabular}{|c|c|c|c|c|}
\hline & $\mathrm{GA}(n=72)$ & Awake $(n=24)$ & Total $(n=96)$ & $\mathrm{p}$ Value \\
\hline \multicolumn{4}{|l|}{ Age, yrs } & \multirow{3}{*}{0.868} \\
\hline Mean (SD) & $57.8(13.9)$ & $58.3(14.8)$ & $57.9(14.1)$ & \\
\hline Range & $28-83$ & $28-83$ & $28-83$ & \\
\hline \multicolumn{4}{|l|}{ Sex, n (\%) } & \multirow{3}{*}{0.900} \\
\hline $\mathrm{F}$ & $23(31.9)$ & $8(33.3)$ & $31(32.3)$ & \\
\hline M & $49(68.1)$ & $16(66.7)$ & $65(67.7)$ & \\
\hline \multicolumn{4}{|l|}{ Race, $n(\%)$} & \multirow{3}{*}{0.735} \\
\hline Asian & $2(2.8)$ & $1(4.2)$ & $3(3.1)$ & \\
\hline White & 70 (97.2) & $23(95.8)$ & $93(96.9)$ & \\
\hline \multicolumn{4}{|l|}{ Diabetes, n (\%) } & \multirow{4}{*}{0.989} \\
\hline No & $52(72.2)$ & $17(70.8)$ & $69(71.9)$ & \\
\hline Insulin-dependent & $6(8.3)$ & $2(8.3)$ & $8(8.3)$ & \\
\hline Non-insulin-dependent & $14(19.4)$ & $5(20.8)$ & $19(19.8)$ & \\
\hline \multicolumn{4}{|l|}{ History of COPD, n (\%) } & \multirow{3}{*}{0.626} \\
\hline No & $68(94.4)$ & $22(91.7)$ & $90(93.8)$ & \\
\hline Yes & $4(5.6)$ & $2(8.3)$ & $6(6.2)$ & \\
\hline \multicolumn{4}{|l|}{ Hypertension, n (\%) } & \multirow{3}{*}{0.512} \\
\hline No & $19(26.4)$ & $8(33.3)$ & $27(28.1)$ & \\
\hline Yes & $53(73.6)$ & $16(66.7)$ & $69(71.9)$ & \\
\hline \multicolumn{4}{|l|}{ ASA class, $n(\%)$} & \multirow{4}{*}{0.992} \\
\hline 1 & $3(4.2)$ & $1(4.2)$ & $4(4.2)$ & \\
\hline ॥ & $47(65.3)$ & $16(66.7)$ & $63(65.6)$ & \\
\hline III & $22(30.6)$ & 7 (29.2) & $29(30.2)$ & \\
\hline \multicolumn{4}{|l|}{ LOS, days } & \multirow{3}{*}{0.002} \\
\hline Mean (SD) & $3.0(2.3)$ & $1.4(1.4)$ & $2.6(2.2)$ & \\
\hline Range & $0-12$ & $0-4$ & $0-12$ & \\
\hline \multicolumn{4}{|l|}{ Op time, hrs } & \multirow{3}{*}{0.247} \\
\hline Mean (SD) & $175.5(123.9)$ & $143.1(96.8)$ & $167.4(118.1)$ & \\
\hline Range & $25-864$ & $45-410$ & $25-864$ & \\
\hline \multicolumn{4}{|l|}{ Discharge, $\mathrm{n}(\%)$} & \multirow{4}{*}{0.507} \\
\hline Home & $68(94.4)$ & $21(87.5)$ & $89(92.7)$ & \\
\hline Rehab & $1(1.4)$ & $1(4.2)$ & $2(2.1)$ & \\
\hline Skilled care facility & $3(4.2)$ & $2(8.3)$ & $5(5.2)$ & \\
\hline \multicolumn{4}{|l|}{ 30-day readmission, $\mathrm{n}(\%)$} & \multirow{3}{*}{0.310} \\
\hline No & $69(95.8)$ & $24(100.0)$ & $93(96.9)$ & \\
\hline Yes & $3(4.2)$ & $0(0.0)$ & $3(3.1)$ & \\
\hline
\end{tabular}

hort. Additional studies aggregating institutional case series, similar to that reported here, will be needed to better resolve these outcomes.

Several centers have reported data on awake spine surgery with early outcomes demonstrating shorter recovery times and improved perioperative morbidity and mortality.,11 Additionally, patient-reported outcomes (PROs) are usually better with the awake spinal fusion protocol. Kolcun et al. reported significant improvement in PROs with no pseudarthrosis or implant failure. ${ }^{11}$ Their results showed that this technique is successful with meaningful improvement in long-term functional status as well as a shorter recovery time, better postoperative analgesia, and less perioperative morbidity and mortality.

One critical benefit to awake TLIFs is conscious sedation, which permits patients to stay conscious and interact with their surgeon throughout intricate steps in the procedure, some of which include manipulating nerve roots that have direct impact on neurological symptoms. ${ }^{7}$ The limited use of intubation and general anesthesia decreases the need for mechanical ventilation, allowing spontaneous breathing to lower intrathoracic pressure and minimize bleeding during procedures. ${ }^{7}$ Wang and Grossman reported their experience after performing endoscopic sin- 
gle-level MIS-TLIF. Ketamine and propofol were used to sedate the patients. Their results showed that arthrodesis rates are successful when compared with open surgery. ${ }^{12}$ While PROs and individual complication rates were not captured in the institutional and national data reported here, these represent avenues for future study.

\section{Limitations}

While our study offers several new insights into outcomes after awake MIS-TLIF based on both institutional and national data, there are several limitations that are important to acknowledge when interpreting the data. First, we could not include radiological findings in our comparisons as these data are not collected by the NSQIP database. Second, patients who had allergies to anesthetic components included in the awake anesthesia protocol could not be assigned to the awake group. Third, a limited amount of data were available in the NSQIP database for awake MIS-TLIF cases, and outcomes in that group may reflect the experience of a few high-performing centers with significant knowledge of the procedure. The NSQIP database does not allow for distinction between MIS versus open TLIF cases and, hence, both were included in our analysis. Lastly, many studies, including our own, that have investigated the outcomes of awake MIS-TLIF have only studied single-level fusions, with very little data available regarding the effects of multilevel fusions.

\section{Conclusions}

Evidence from our institutional experience and national analysis demonstrates that awake MIS-TLIF can be performed safely with no major perioperative complications and may reduce LOS compared with cases performed under general anesthesia.

\section{References}

1. Hammad A, Wirries A, Ardeshiri A, Nikiforov O, Geiger F. Open versus minimally invasive TLIF: literature review and meta-analysis. J Orthop Surg Res. 2019;14(1):229.

2. de Kunder SL, van Kuijk SMJ, Rijkers K, Caelers IJMH, van Hemert WLW, de Bie RA, van Santbrink H. Transforaminal lumbar interbody fusion (TLIF) versus posterior lumbar interbody fusion (PLIF) in lumbar spondylolisthesis: a systematic review and meta-analysis. Spine J. 2017;17(11):1712-1721.

3. Kim JE, Choi DJ. Biportal endoscopic transforaminal lumbar interbody fusion with arthroscopy. Clin Orthop Surg. 2018; 10(2):248-252.

4. Ge DH, Stekas ND, Varlotta CG, Fischer CR, Petrizzo A, Protopsaltis TS, et al. Comparative analysis of two transforaminal lumbar interbody fusion techniques: open TLIF versus Wiltse MIS TLIF. Spine (Phila Pa 1976). 2019;44(9):E555-E560.
5. Foley KT, Holly LT, Schwender JD. Minimally invasive lumbar fusion. Spine (Phila Pa 1976). 2003;28(15)(suppl): S26-S35.

6. De Biase G, Bechtle P, Leone B, Quinones-Hinojosa A, Abode-Iyamah K. Awake minimally invasive transforaminal lumbar interbody fusion with a pedicle-based retraction system. Clin Neurol Neurosurg. 2021;200:106313.

7. Kai-Hong Chan A, Choy W, Miller CA, Robinson LC, Mummaneni PV. A novel technique for awake, minimally invasive transforaminal lumbar interbody fusion: technical note. Neurosurg Focus. 2019;46(4):E16.

8. Kolcun JPG, Brusko GD, Wang MY. Endoscopic transforaminal lumbar interbody fusion without general anesthesia: technical innovations and outcomes. Ann Transl Med. 2019; 7(5)(suppl 5):S167.

9. Garcia RM, Khanna R, Dahdaleh NS, Cybulski G, Lam S, Smith ZA. Thirty-day readmission risk factors following single-level transforaminal lumbar interbody fusion (TLIF) for 4992 patients from the ACS-NSQIP database. Global Spine J. 2017;7(3):220-226.

10. De Biase G, Chen S, Akinduro O, Quinones-Hinojosa A, Abode-Iyamah K. Awake robotic minimally invasive L4-5 transforaminal lumbar interbody fusion. World Neurosurg. 2021;148:93.

11. Kolcun JPG, Brusko GD, Basil GW, Epstein R, Wang MY. Endoscopic transforaminal lumbar interbody fusion without general anesthesia: operative and clinical outcomes in 100 consecutive patients with a minimum 1-year follow-up. Neurosurg Focus. 2019;46(4):E14.

12. Wang MY, Grossman J. Endoscopic minimally invasive transforaminal interbody fusion without general anesthesia: initial clinical experience with 1-year follow-up. Neurosurg Focus. 2016;40(2):E13.

\section{Disclosures}

Dr. Abode-Iyamah: consultant for Stryker.

\section{Author Contributions}

Conception and design: Abode-Iyamah, Ghaith, Bhandarkar, Rajjoub. Acquisition of data: Abode-Iyamah, Ghaith, Bhandarkar, De Biase. Analysis and interpretation of data: Ghaith. Drafting the article: Ghaith, Rajjoub. Critically revising the article: Bydon, Abode-Iyamah, De Biase, Quiñones-Hinojosa. Reviewed submitted version of manuscript: Bydon, Abode-Iyamah, De Biase, Quiñones-Hinojosa. Approved the final version of the manuscript on behalf of all authors: Bydon. Statistical analysis: Ghaith, Bhandarkar, Rajjoub. Study supervision: Bydon, Abode-Iyamah, Ghaith, Chen, Quiñones-Hinojosa.

\section{Correspondence}

Mohamad Bydon: Mayo Clinic, Rochester, MN. bydon.mohamad@mayo.edu. 